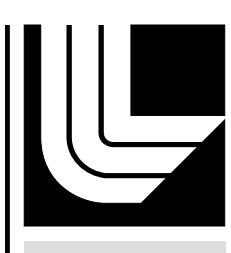

LAWRENCE LIVERMORE NATIONAL LABORATORY

Enhanced Energetic Stability and Optical Activity of Symmetry-Reduced C60

M. R. Manaa

January 25, 2006

Chemical Physics Letters 
This document was prepared as an account of work sponsored by an agency of the United States Government. Neither the United States Government nor the University of California nor any of their employees, makes any warranty, express or implied, or assumes any legal liability or responsibility for the accuracy, completeness, or usefulness of any information, apparatus, product, or process disclosed, or represents that its use would not infringe privately owned rights. Reference herein to any specific commercial product, process, or service by trade name, trademark, manufacturer, or otherwise, does not necessarily constitute or imply its endorsement, recommendation, or favoring by the United States Government or the University of California. The views and opinions of authors expressed herein do not necessarily state or reflect those of the United States Government or the University of California, and shall not be used for advertising or product endorsement purposes. 


\title{
Enhanced Energetic Stability and Optical Activity of Symmetry-Reduced $\mathrm{C}_{60}$
}

\author{
M. Riad Manaa
}

University of California, Lawrence Livermore National Laboratory, Energetic Materials Center, P.O. Box 808, L282, Livermore, California, 94551

Manaa1@1lnl.gov

RECEIVED DATE (will be automatically inserted after manuscript is accepted)

Since its discovery in $1985,{ }^{1}$ the celebrated geodesic cage structure of the $\mathrm{C}_{60}$ molecule has been recognized: ${ }^{2}$ a truncated icosahedron in which all sixty vertices are equivalent and has the full $\mathrm{I}_{h}$ symmetry, making it thus far the most spherical of all known molecules. Inherent in this high symmetry is an intricate network of electron-phonon coupling, evident in phonon progressions and vibronic peak broadening, ${ }^{3}$ and resulting in structural distortions of neutral $\mathrm{C}_{60}$ in the presence of solvent. ${ }^{4-6}$ Within the $\mathrm{I}_{h}$ symmetry group of this molecule, of the forty-six distinct vibrational frequencies only ten are Raman-active and four are IR-active (in the first order), while the remaining 32 modes are optically silent. ${ }^{7}$ Symmetryreduced structures of $\mathrm{C}_{60}$ would activate some of these silent modes, which could then be amenable to experimental verification such as in resonance Raman scattering. ${ }^{6,8-9}$ Here, quantum chemical calculations within density functional theory establish for the first times (1) lower-symmetry, energetically more stable structures for $\mathrm{C}_{60}$, the lowest of which is of $\mathrm{D}_{3 d}$ symmetry, and with a new assignment of the ground state as the ${ }^{1} \mathrm{~A}_{l g}$ state, (2) the activation of some IR and Raman $\mathrm{I}_{h}$ silent modes: the IR $\mathrm{H}_{u}$ mode around $540 \mathrm{~cm}^{-1}$ and $\mathrm{G}_{u}$ band at $1465 \mathrm{~cm}^{-}$ ${ }^{1}$, and the Raman $\mathrm{G}_{g}$ mode around $1530 \mathrm{~cm}^{-1}$.

Several quantum chemical calculations at the density functional B3LYP and BPW91 levels, ${ }^{10-14}$ and using two different basis sets (Table 1) were performed for symmetry constrained structures of $\mathrm{C}_{60}: \mathrm{I}_{h}, \mathrm{~T}_{h}, \mathrm{D}_{5 d}, \mathrm{D}_{3 d}$, and $\mathrm{S}_{6}$. Optimization within these symmetry constraints proved, consistently, the $\mathrm{D}_{3 d}$ structure to be the lowest energy conformation at all levels of theory, being $0.8-0.9 \mathrm{kcal} / \mathrm{mol}$ lower than the $\mathrm{I}_{h}$ symmetry molecule, as listed in Table 1.



Figure 1. B3LYP/6-31G* optimized, symmetry - unique $\mathrm{D}_{3 d}$ carboncarbon bonds of $\mathrm{C}_{60}$ (in $\AA$ ). $\mathrm{X}$ denotes the $\mathrm{C}_{3}$ axis of symmetry.

Table 1. Energetic of symmetry-constraints structures of $\mathrm{C}_{60}$ at various levels of theory.

\begin{tabular}{|c|c|ccc|}
\hline \multirow{2}{*}{ Method } & Total Energy & \multicolumn{3}{|c|}{ Relative Energy $^{\mathrm{a}}$} \\
& $\mathrm{I}_{h}$ & $\mathrm{D}_{3 d}$ & $\mathrm{D}_{5 d}$ & $\mathrm{~S}_{6}$ \\
\hline B3LYP/6-31G* & -2286.173080 & -0.8 & -0.6 & -0.7 \\
\hline B3LYP/cc-pVDZ & -2286.314164 & -0.9 & -0.7 & -0.7 \\
\hline BPW91/6-31G* & -2286.069710 & -0.9 & -0.7 & -0.7 \\
\hline
\end{tabular}

a in $\mathrm{Kcal} / \mathrm{mol}$.

Figure 1 shows the optimized unique bonds of the lowest energy $\mathrm{D}_{3 d}$ symmetric $\mathrm{C}_{60}$ at the B3LYP/6-31G* level of computation. Within this symmetry, there are 10 unique carbon-carbon bonds. Optimization of the $\mathrm{I}_{h}$ structure at the same level yields 1.454 and $1.396 \AA$ for the two unique C-C bonds, to be compared with neutron scattering average values of 1.391 and $1.455 \AA .{ }^{15}$ Note that the diffraction results are averages for three sets of values comprising of fifteen measured bonds. The $\mathrm{D}_{3 d}$ optimized structure has four short bonds in the range 1.396-1.410 $\AA$, and six long bonds within the range 1.447-1.459 $\AA$. These values illustrate only small distortions from the $\mathrm{I}_{h}$ structure, consistent with the slight energy change reported in Table 1 . The $\mathrm{C}_{60}$ molecule transitions from a spherical top form in the $\mathrm{I}_{h}$ symmetry to a prolate symmetric top form in the $\mathrm{D}_{3 d}$ symmetry structure. This symmetry lowering produces a slight, anisotropic change in the quadrupole moment from $\sigma=-324.09$ (Debye- $\AA$ ) in $\mathrm{I}_{h}$ symmetry, to $\sigma_{\mathrm{XX}}=\sigma_{\mathrm{YY}}=-324.09, \sigma_{\mathrm{ZZ}}=-324.15$ in the $\mathrm{D}_{3 d}$ structure. It should be noted that a change in the quadrupole moment has been predicted by Gallagher et al. in order to account for their observed solvatochromism shifts of $\mathrm{C}_{60} \cdot{ }^{16}$

The energetic of four symmetry-reduced structures at various level of treatment are listed in Table 1 , excluding the $\mathrm{T}_{h}$ symmetry structure, which was energetically identical to the $\mathrm{I}_{h}$ conformation. All three structures of $\mathrm{D}_{3 d}, \mathrm{D}_{5 d}$, and $\mathrm{S}_{6}$ are almost degenerate and lower in energy than the $\mathrm{I}_{h}$ structure by as much as $0.9 \mathrm{Kcal} / \mathrm{mol}(49 \mathrm{meV})$ for the $\mathrm{D}_{3 d}$ structure. Reorganization among these various symmetry structures can thus occur easily through tunneling or due to external perturbations such as solvent environment. It is interesting to note that similar $\mathrm{D}_{3 d}$ and $\mathrm{D}_{5 d}$ lower energy structures; Jahn-Teller distortions in the 
$\mathrm{C}_{60}$ anion radical have been predicted. ${ }^{17}$ Transitions between neutral and anionic $\mathrm{C}_{60}$, both of which are now geometrically of $\mathrm{D}_{3 d}$ symmetry, is very favorable according to the FranckCondon principle. Further, with the full vibrational structure of $\mathrm{I}_{h}, \mathrm{D}_{3 d}$, and $\mathrm{D}_{5 d}$ symmetry calculated, the $\mathrm{D}_{3 d}$ structure has the largest zero-point energy of $235.3 \mathrm{kcal} / \mathrm{mol}$, compared with $234.7 \mathrm{kcal} / \mathrm{mol}$ for the $\mathrm{I}_{h}$ structure at the B3LYP/6-31G* level.

A consequence of symmetry lowering is the splitting of electronic molecular energy levels. The highest occupied state of $\mathrm{C}_{60}$ in $\mathrm{I}_{h}$ symmetry is the completely occupied, five-fold degenerate $h_{u}$ state, while the lowest unoccupied state is the three- fold degenerate $t_{l u}$ state. The energy band gap between the HOMO and LUMO states is calculated to be $2.76 \mathrm{eV}$ at the B3LYP/6-31G* level. In $\mathrm{D}_{3 d}$ symmetry, the $\mathrm{h}_{u}$ state splits into one $\mathrm{a}_{2 u}$ and two doubly degenerate $\mathrm{e}_{u}$ states, separated by $24 \mathrm{~cm}^{-}$ ${ }^{1}$. The $\mathrm{t}_{l u}$ LUMO state splits into $\mathrm{a}_{2 u}$ and $\mathrm{e}_{u}$, with the $\mathrm{a}_{2 u}$ level being $18 \mathrm{~cm}^{-1}$ lower in energy at this level of calculation. The HOMO-LUMO separation is then an $\mathrm{e}_{u}-\mathrm{e}_{u}$ gap in $\mathrm{D}_{3 d}$ symmetry, equal to that of the $I_{h}$ symmetry. The level splitting in this symmetry causes both the HOMO and LUMO to be shifted higher in energy by an almost similar magnitude.

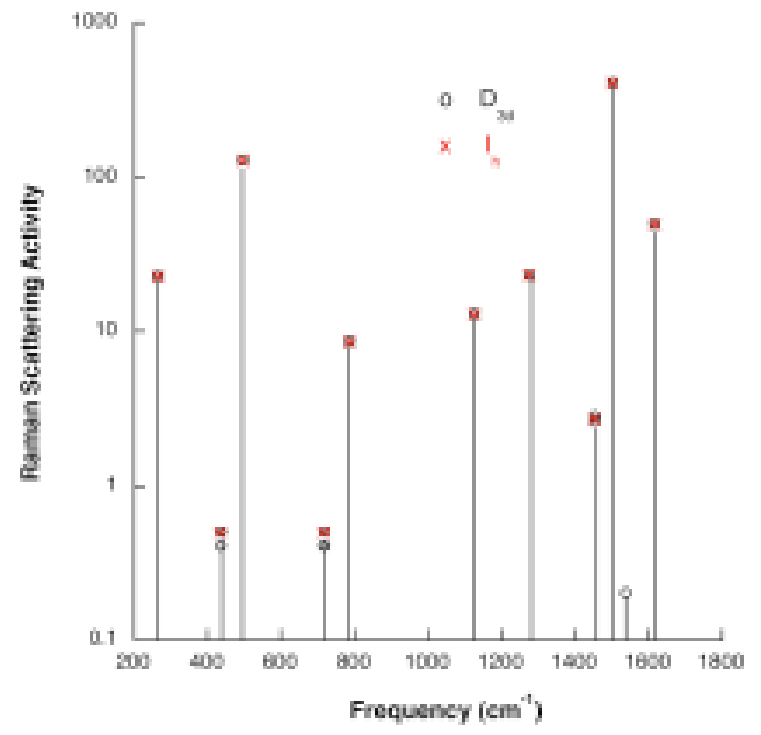

Figure 2. Calculated Raman scattering activities (in $\AA^{4} / a . m . u$ ) of $\mathrm{D}_{3 d}$ and $\mathrm{I}_{h}$ symmetry structures of $\mathrm{C}_{60}$.

The calculated Raman scattering activities of for the $I_{h}$ and $\mathrm{D}_{3 d}$ symmetry structures at the B3LYP/6-31G* level is shown in Figure 2. In $\mathrm{I}_{h}$ symmetry, two $\mathrm{A}_{g}$ and eight $\mathrm{H}_{g}$ fundamental vibrational modes are Raman active, altogether with combination and overtone modes have been experimentally assigned for the solid phase. ${ }^{18}$ The calculated and measured frequencies are in good agreement. In $\mathrm{D}_{3 d}$ symmetry, the $\mathrm{H}_{g}$ mode correlates with one $\mathrm{A}_{l g}$ and two $\mathrm{E}_{g}$ modes. The calculated Raman activities show very small splitting of the active modes: the lowest frequency $\mathrm{H}_{\mathrm{g}}$ mode at $265 \mathrm{~cm}^{-1}$ splits into two modes at 266 and $267 \mathrm{~cm}^{-1}$. Similar splitting for all other active modes at 434, 715, 786, 1125, 1275,1453 , and $1616 \mathrm{~cm}^{-1}$, with a frequency shift of at most 3 $\mathrm{cm}^{-1}$ is calculated for the $\mathrm{D}_{3 d}$ symmetry structure. Similar results have been obtained for the calculated Raman activities of the $\mathrm{D}_{5 d}$ structure, for which the $\mathrm{H}_{g}$ mode splits into the $\mathrm{A}_{1 g}, \mathrm{E}_{1 g}$ and $\mathrm{E}_{2 g}$ modes. For this structure, the only active modes in the region of up to $500 \mathrm{~cm}^{-1}$ are determined at 266, 436, and 497 $\mathrm{cm}^{-1}$. Thus, it is concluded that a recent claim attributing the appearance of a resonance Raman band at $281 \mathrm{~cm}^{-1}$ is primarily due to distortion to either $\mathrm{D}_{3 d}$ or $\mathrm{D}_{5 d}$ structures of $\mathrm{C}_{60}$ in solution is precluded. ${ }^{6}$

The Raman spectrum of $\mathrm{D}_{3 d} \mathrm{C}_{60}$ in figure 2 shows a weak band at $1539 \mathrm{~cm}^{-1}$. This is due to the activation of otherwise silent mode, and of $\mathrm{A}_{1 g}$ character. Two much weaker $\mathrm{E}_{g}$ modes also appear at $1538 \mathrm{~cm}^{-1}$. Experimentally, a silent $\mathrm{G}_{g}$ band at 1525 $\mathrm{cm}^{-1}$ has been assigned in $\mathrm{I}_{h}$ symmetry. In $\mathrm{D}_{3 d}$ symmetry, this mode should correlate with the $\mathrm{A}_{1 g}, \mathrm{~A}_{2 g}$, and $\mathrm{E}_{g}$ modes, of which only the $\mathrm{A}_{l g}$ and $\mathrm{E}_{g}$ are Raman active. The appearance of this band in a region significantly removed from other fundamentals should, therefore, be a Raman signature for the existence of this structure and could be discerned in highresolution Raman spectroscopy of $\mathrm{C}_{60}$ in the gas-phase. The existence of two $\mathrm{H}_{g}$ overtones in the same region, however, could prove its identification a challenging task indeed. ${ }^{18}$

The four IR-active $\mathrm{T}_{1 u}$ modes in the $\mathrm{I}_{h}$ symmetry are calculated at the B3LYP/6-31G* level to have frequencies of $532,587,1212$, and $1459 \mathrm{~cm}^{-1}$. These also compare well with the experimental measurements of 527, 576, 1183, and 1430 $\mathrm{cm}^{-1}$. ${ }^{19}$ The small distortions into $\mathrm{D}_{3 d}$ symmetry shift these frequencies to higher values of an utmost $5 \mathrm{~cm}^{-1}$. Two weak bands are activated: one with frequencies of $541,543 \mathrm{~cm}^{-1}$, and a weaker still band at $1465 \mathrm{~cm}^{-1}$. The first could be attributed to the $\mathrm{I}_{h} \mathrm{H}_{u}$ silent mode at the experimentally assigned 563 band, while the second emanate from the silent $G_{u}$ band, appearing with a frequency of $1446 \mathrm{~cm}^{-1} .{ }^{19}$ Previous assignments of weak IR modes, specifically in the high frequency region, as due to combination modes caused by anharmonicity may well in fact be originated from a geometrical change of $\mathrm{C}_{60}$ to the lower $\mathrm{D}_{3 d}$ symmetry. ${ }^{20}$

The present work shows that small distortions from the perfectly spherical $\mathrm{C}_{60}$ to lower symmetries are energetically feasible. Activations of otherwise silent IR and Raman modes may perhaps require the assignments of previously identified combinations modes as fundamentals in the new, lowest energy $\mathrm{D}_{3 d}$ symmetry structure.

\section{Acknowledgment.}

This work was performed under the auspices of the U.S. Department of Energy by UC, Lawrence Livermore National Laboratory under contract number W-7405-Eng-48.

REFERENCES

(1) Kroto, H. W.; Heath, J. R.; O'Brien, S. C.; Curl, R. F.; Smalley, R. E. Nature 1985, 318, 162.

(2) Kroto, H. W.; Allaf, A. W.; Balm, S. P. Chem. Rev. 1991, 91, 1213

(3) Reber, C.; Yee, L.; McKiernan, J.; Zink, J. I.; Williams, R. S.; Tong, W. M.; Ohlberg, D. A.; Whetten, R. L.; Diederich, F. J. Phys. Chem. 1991, $95,2127$.

(4) Rubstov, I. V.; Khudiakov, D. V.; Nadtochenko, V. A.; Lobach, A. S.; Moravskii, A. P. Chem. Phys. Lett. 1994, 229, 517.

(5) Gallagher, S. H.; Armstrong, R. S.; Lay, P. A.; Reed, C. A. Chem. Phys. Lett. 1996, 248, 353.

(6) Gallagher, S. H.; Thompson, K. C.; Armstrong, R. S.; Lay, P. A. J. Phys. Chem. A 2004, 108, 5564.

(7) Dresselhaus, M. S.; Dresselhaus, G.; Eklund, P. C. Science of Fullerenes and Carbon Nanotubes, 1996, Academic Press Inc., San Diego, USA. Chapter 11.

(8) Gallagher, S. H.; Armstrong, R. S.; Lay, P. A.; Reed, C. A. J. Am. Chem. Soc. 1994, 116, 12091.

(9) Gallagher, S. H.; Armstrong, R. S.; Clucas, W. A.; Lay, P. A.; Reed, C. A. J. Phys. Chem. A 1997, 101, 2960.

(10) Becke, A. D. J. Chem. Phys. 1993, 98, 5648.

(11) Lee, C.; Yang, W.; Parr, R. G. Phys. Rev. B 1988, 37, 785.

(12) Becke, A. D. Phys. Rev. A 1988, 38, 3098.

(13) Perdew, J. P.; Wang, Y. Phys. Rev. B 1992, 45, 13244. 
(14) Frisch, M. J.; et al., Gaussian 98, Rev. A.4, 1998, Gaussian, Inc., Pittsburgh PA, USA.

(15) David, W. I. F.; Ibberson, R. M.; Matthewman, J. C.; Prassides, K.; Dennis, T. J. S.; Hare, J. P.; Kroto, H. W.; Taylor, R.; Walton, D. R. M. Nature 1991, 353, 147.

(16) Gallagher, S. H.; Armstrong, W. A.; Lay, P. A.; Reed, C. A. J. Phys. Chem. 1995, 99, 5817.

(17) Koga, N.; Morokuma, K. Chem. Phys. Lett. 1992, 196, 191.

(18) Dong, Z. H.; Zhou, P.; Holden, J. M.; Eklund, P. C.; Dresselhaus, M. S.; Dresselhaus, G. Phys. Rev. B 1993, 48, 2862.

(19) Wang, K. A.; Rao, A. M.; Eklund, P. C.; Dresselhaus, M. S.; Dresselhaus, G. Phys. Rev. B 1993, 48, 11375 .

(20) Fabian, J. Phys. Rev. B 1996, 53, 13864. 
Graphic entry for the Table of Contents (TOC)

\section{Abstract:}

Quantum chemical calculations within density functional theory establish that small distortions from the perfectly spherical $I_{h}$ symmetry of $C_{60}$ to lower symmetries are energetically feasible. $A D_{3 d}$ symmetry structure proved to be the lowest energy structure, almost $0.9 \mathrm{kcal} / \mathrm{mol}$ lower than the $I_{h}$ symmetry structure. Activations of otherwise $I_{h}$ symmetrical silent modes, the IR $G_{u}$ band at $1465 \mathrm{~cm}^{-1}$, and the Raman $\mathrm{G}_{g}$ mode around $1530 \mathrm{~cm}^{-1}$ may require the assignments of previously identified combinations modes as fundamentals in the new symmetry structure. A recent experimental observation that attribute the appearance of a resonance Raman band at $281 \mathrm{~cm}^{-1}$ as primarily due to distortion to either $\mathrm{D}_{3 d}$ or $\mathrm{D}_{5 d}$ structures of $\mathrm{C}_{60}$ in solution is discounted.

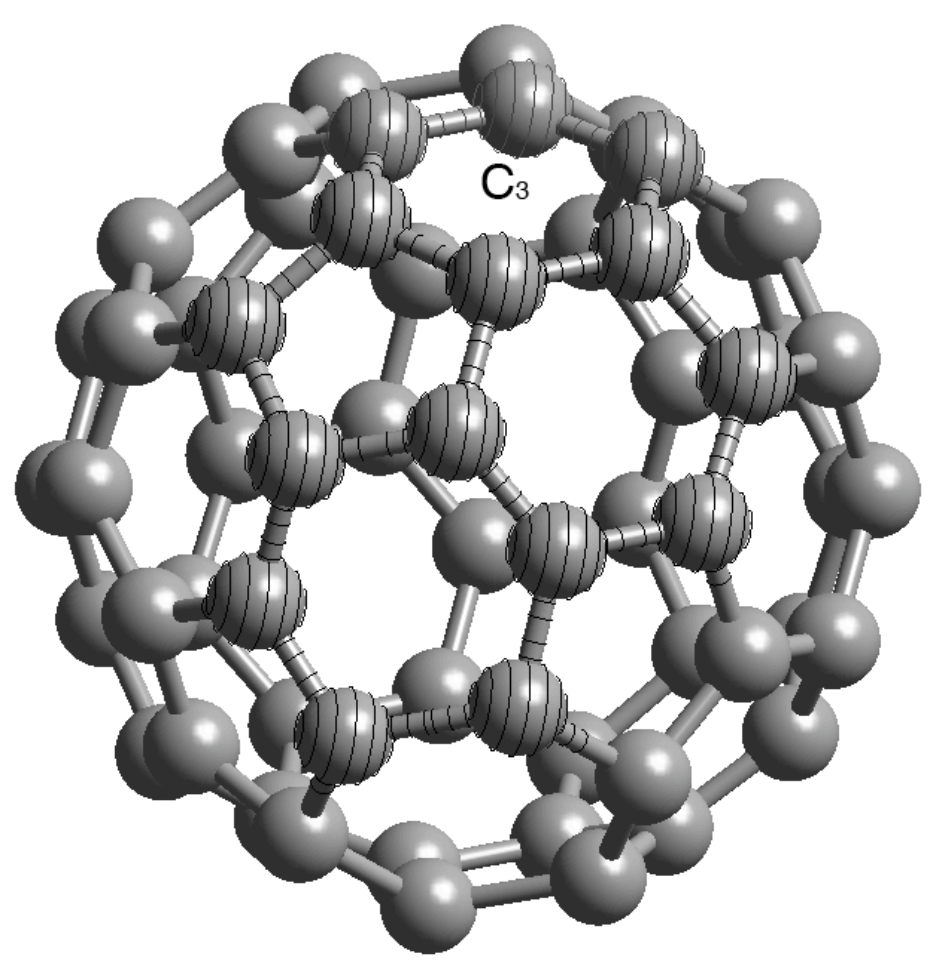

